\title{
LES QUALITÉS DU CONSEILLER: SAVOIR, BIENVEILLANCE, FRANC-PARLER (PLATON, GORGAS, 486 E 6-487 D 7)
}

\author{
Annie Hourcade \\ Université de Rouen (Haute-Normandie)/ERIAC/IHP
}

\begin{abstract}
The Gorgias plays a main part to precise the Socratic and Platonician conception of the adviser (sumboulos). The qualities which Socrates attributes, ironically, to Callicles: knowledge (epistêmê), good will (eunoia) and frank speech (parrhêsia) must be considered as the qualities of the philosopher in so far as he is the authentic adviser. As a matter of fact, these qualities are opposed to the characteristics of the rhetor's and the public man's souls: skillful in conjecturing (stokhastikê), manly (andreia) and naturally tough in human relations (phusei deinê prosomilein tois anthrôpois), characteristics which Callicles brings into contrast with the portrait he paints of Socrates, as a man of privacy, lacking manliness. This passage of the Gorgias finds, besides, an echo in Aristotle's treatises: the Rhetoric (book II) and the Nicomachean Ethics (book VI).

Keywords: Plato, Gorgias, knowledge, epistêmê, good will, eunoia, frank speech, parrhêsia, adviser, sumboulos, Aristotle, Rhetoric, Nicomachean Ethics
\end{abstract}

Resumo: O Górgias representa um momento essencial no sentido de precisar a concepção Socrática e Platônica do conselheiro (sumboulos). As qualidades que Sócrates atribui, ironicamente, a Cálicles: conhecimento (epistêmê), boa vontade (eunoia) e discurso franco (parrhêsia), devem ser consideradas como as qualidades dos filósofos na medida em que ele é o conselheiro autêntico. Como assunto de fato, estas qualidades são opostas às características da alma do orador e do homem público: hábil em conjeturar (stokhastikê), viril (andreia) e naturalmente agressivo nas relações humanas (phusei deinê prosomilein tois anthrôpois), características que Cálicles contrasta com o quadro que pinta de Sócrates, como um homem de privacidade, carente de virilidade. Esta passagem do Górgias encontra eco, além disto, nos trtados de Aristóteles: a Retórica (livro II) e a Ética Nicomaquéia (livro VI).

Palavras-chave: Platão, Górgias, conhecimento, epistêmê, boa vontade, eunoia, discurso franco, parrhêsia, conselheiro, sumboulos, Aristóteles, Retórica, Ética Nicomaquéia. 


\section{Introduction}

Le but de cette étude est de s'interroger sur un passage du Gorgias de Platon dans le cadre duquel Socrate, après avoir entendu Calliclès lui conseiller d'abandonner le mode de vie philosophique, affirme que c'est pour lui, Socrate, une véritable aubaine de l'avoir rencontré, au même titre que c'est une véritable aubaine de trouver ce que l'on nomme "pierre de touche", pierre qui a la propriété de détecter l'or. Calliclès, en effet, pour Socrate, peut être assimilé à une pierre de touche dans la mesure où il présente trois caractéristiques: le savoir (epistêmê), la bienveillance (eunoia) et le francparler (parrhêsia) qui le rendent apte à mettre l'âme de Socrate à l'épreuve et déterminer si elle est d'or, en d'autres termes, si elle adopte la meilleure façon de vivre. Le propos est vraisemblablement ironique ${ }^{1}$. Il est en rupture, en effet, nous le verrons, avec tout ce qui précède, en rupture avec les caractéristiques de l'âme du rhéteur, de l'homme public, habile à conjecturer (stokhastikê), virile (andreia) et naturellement redoutable dans les rapports

\footnotetext{
1 L'enjeu n'est pas, ou pas exclusivement, pour Socrate, lorsqu'il décrit ironiquement l'âme de Calliclès comme dotée de science, de bienveillance et de franc-parler, de mener un combat ad hominem - même s'il le fait, sans aucun doute, ailleurs et d'une autre façon dans son échange avec Calliclès, lorsqu'il confronte leurs conceptions de la vie telle qu'elle doit être vécue (492 d 3-5) et telle que l'un et l'autre la vivent; sur ce point voir KAHN, CH. H. Drama and Dialectic in Plato's Gorgias. Oxford Studies in Ancient Philosophy, 1, pp. 75-121, 1983, particulièrement pp. 75-76 et 110. II s'agit aussi pour Socrate d'opposer, de manière plus générale, philosophes et rhéteurs sur ce terrain qui, plus que tout autre, leur est commun: le conseil. C'est en cela que l'enjeu du passage sur les qualités du conseiller dépasse les limites du débat avec Calliclès en nous invitant à garder à l'esprit les analyses menées, dans le cadre du dialogue avec Gorgias, sur la rhétorique qui use de persuasion de croyance et non d'enseignement. Sur la thèse selon laquelle il existe des affinités entre Platon et Calliclès, ce qui atténue la dimension ironique du passage sans pour autant rendre caduque l'affirmation selon laquelle epistêmê, eunoia, parrhêsia sont les qualités du philosophe, voir l'introduction que E. R. Dodds propose à son édition du Gorgias, notamment pp. 13-14: « Plato had certainly known such men in his early youth, and had probably felt a measure of sympathy for them. He admired their candour, and he shared their contempt for the masses, for the professors of aretê, and for all the hypocrisies of a society whose morality was built on appearances [...] Hence his portrait of Callicles not only has warmth and vitality but is tinged with a kind of regretful affection », DODDS, E. R. Plato. Gorgias. A Revised Text with Introduction and Commentary. Oxford: Clarendon Press, 1959; WOOLF, R. Callicles and Socrates: Psychic (Dis)harmony in the Gorgias. Oxford Studies in Ancient Philosophy, 18, pp. 1-40, 2000; VAN RAALTE M. Socratic Parrhesia and its Afterlife in Plato's Laws. In SLUITER, I., ROSEN, R. M., Free Speech in Classical Antiquity. Leiden-Boston: Brill, pp. 279-312, 2004, particulièrement pp. 286-288. Voir également les arguments décisifs que Richard McKim propose pour une lecture du passage comme représentatif de l'ironie socratique: MCKIM, R. Shame and Truth in Plato's Gorgias. In GRISWOLD, CH. L. Platonic Writings. Platonic Readings. New York-London: Routledge, pp. 34-48, 1988, tout particulièrement pp. 40-41.
} 
humains (phusei deinê prosomilein tois anthrôpois), caractéristiques réaffirmées par Calliclès par contraste avec ce portrait-charge qu'il brosse de Socrate, comme un homme du privé, dénué de toute forme de courage; mais le passage est aussi en rupture avec la suite du dialogue, de fait, c'est Socrate qui en viendra, par divers moyens - dialogue, monologue puis recours au mythe -, à conseiller à Calliclès de vivre en philosophe ${ }^{2}$. Epistêmê, eunoia, parrhêsia peuvent donc être appréhendées comme les qualités emblématiques $\mathrm{du}$ philosophe $\mathrm{e}^{3}$, tel que le conçoit Platon, dans sa dimension authentiquement conseillère ${ }^{4}$.

La portée du passage étudié dépasse cependant les limites du Gorgias dans la mesure où il trouve deux échos précis chez Aristote, d'une part, dans un passage de la Rhétorique, passage qui porte, lui aussi, sur le conseil et reprend non seulement une partie des qualités du conseiller, mais aussi la construction même du passage du Gorgias, introduisant néanmoins une conception du conseil fondamentalement différente de celle que Platon, dans le Gorgias, fait sienne; d'autre part, dans les développements qu'Aristote

\footnotetext{
2 Même si le conseil de Socrate, en définitive, se solde, dans le cas précis de Calliclès, par un échec. Les raisons pour lesquelles Socrate échoue ont donné lieu à de nombreuses études. Voir notamment, BEVERSLUIS, J. Cross-Examining Socrates. A Defense of the Interlocutors in Plato's Early Dialogues. Cambridge: Cambridge University Press, 2000, p. 339, qui fait remarquer que Calliclès est l'unique interlocuteur que Socrate ne déclare pas avoir réfuté. Dans le cadre de la problématique du conseil, cependant, on peut poser l'hypothèse que Calliclès, s'il ne possède pas les qualités requises pour prétendre donner d'authentiques conseils, témoigne en outre d'une inaptitude foncière à en recevoir. Cf. sur ce point, Platon, Lois, IV, 709 d 7-8; Lettre VII, 327 a 6-b 1.

3 Sur ce point, voir MCCOY, M. Plato on the Rhetoric of Philosophers and Sophists. Cambridge: Cambridge University Press, 2008, p. 87 qui souligne, dans ses développements sur epistêmê, eunoia, parrhêsia, que dans le Gorgias, "philosophical rhetoric is distinguished from sophistic rhetoric not by a precise method but rather by how Socrates' speech is informed by the presence of these key virtues », voir également, dans le même ouvrage, pp. 103-110.

${ }^{4}$ Le problème du rapport que Socrate et Platon entretiennent avec la politique et, plus précisément, avec la démocratie, dépasse le cadre de cette étude. On peut cependant souligner les limites d'une conception des dialogues platoniciens comme emblématiques d'une forme de démocratie délibérative. II est vrai qu'un dialogue comme le Gorgias, qui valorise le franc-parler et le consensus, peut inciter à soutenir une telle thèse. C'est le cas notamment de EUBEN, J. P. Reading Democracy: "Socratic" Dialogues and the Political Education of Democratic Citizens. In: OBER, J., HEDRICK, C. Dêmokratia. Princeton: Princeton University Press, pp. 327-359, 1996. Voir également la critique que Malcolm Schofield propose de cette perspective: SCHOFIELD, M. Plato. Political Philosophy. Oxford: Oxford University Press, 2006, pp. 5559, ainsi que l'article de STALLEY, R. F. The Politics of the Gorgias. In: ERLER, M., BRISSON, L. Gorgias - Menon, Selected Papers from the Seventh Symposium Platonicum. Sankt Augustin: Academia Verlag, pp. 116-121, 2007, qui considère (p. 120) que le Gorgias semble être plus anti-politique qu'antidémocratique. De fait, l'apparente incompatibilité entre un Socrate qui se décrit lui-même comme un homme du privé et qui déclare pourtant être le seul à s'occuper authentiquement des affaires de l'État (521 d 6-8) se trouve ainsi être levée et contribue à distinguer clairement le conseil, tel que l'entend Socrate, et le conseil tel que le pratiquent sophistes et rhéteurs. Sur ce point, cf. également infra n. 11.
} 
propose, au livre VI de l'Éthique à Nicomaque, sur l'euboulia (le bon conseil) qui n'est ni epistêmê (science), ni eustokhia (intuition juste), passage dont le but est de repenser et de préciser la conception platonicienne du conseil, telle qu'elle est développée, tout particulièrement, dans le Gorgias.

\section{La place du conseil dans le Gorgias}

Le Gorgias est un dialogue dans lequel Platon aborde, de manière privilégiée, la notion de conseil. Le fait de donner des conseils (sumbouleuein) constitue l'activité dont le rhéteur revendique l'exercice comme emblématique de la puissance de son art. C'est précisément en matière de conseil - et non bien sûr en matière de production ou de fabrication - que le rhéteur peut rivaliser avec les professionnels, qu'ils soient médecins, architectes ou constructeurs de navire. Le conseil, en ce sens, structure un passage important du début du Gorgias (455 b-456 c), passage dans lequel Gorgias a, sans aucun doute, au moins provisoirement, l'avantage sur Socrate, précisément en raison de cette référence, renouvelée, au conseil, qui aboutira à la conclusion selon laquelle la rhétorique, telle que l'entend Gorgias, est bien un art. Ainsi, après que Socrate a ménagé la différence entre la persuasion de croyance, par définition susceptible d'être vraie ou fausse, dont relève la rhétorique de Gorgias, et une rhétorique de persuasion d'enseignement, fondée sur un savoir (epistêmê) et donc nécessairement vraie, Socrate introduit lui-même la notion de conseil qu'il présente d'emblée comme affaire de spécialiste (dêmiourgos), le rhéteur, au contraire, devant se voir refuser, selon lui, le droit de donner des conseils (455 b 2-5): "Quand une assemblée de citoyens se forme pour choisir des médecins, des constructeurs de navires ou tout autre de la gent des professionnels (dêmiourgikou ethnous), n'est-il pas vrai qu'alors, ce ne sera pas à celui qui est compétent en rhétorique (rhêtorikos) de donner des conseils (ou sumbouleuser)? Il est évident, en effet, dans chacun de ces choix, qu'il faut choisir le plus compétent (ton tekhnikôtaton)". C’est donc bien celui qui est le plus apte à mettre en œuvre une tekhnê, à produire, par conséquent, ce qu'en tant que spécialiste son art lui permet de produire: la santé, le navire etc., qui seul peut légitimement, pour Socrate, donner des conseils. Le 
conseiller dans un domaine donné sera donc nécessairement un technicien compétent dans ce même domaine ${ }^{5}$.

C'est pourtant cette référence socratique au conseil qui va montrer le chemin à Gorgias, comme ce dernier le souligne lui-même (455 d 7-8), afin de dévoiler à Socrate toute l'efficacité de la rhétorique. Il lui suffit pour cela de s'appuyer sur les faits eux-mêmes: "Les arsenaux [d'Athènes], les murs, l'aménagement des ports, les Athéniens les doivent, pour partie aux conseils (sumboulês) de Thémistocle, pour partie, à ceux de Périclès, mais pas aux professionnels (dêmiourgôn)" (455 d 8-e 3). Socrate, d'ailleurs, en convient pour avoir lui-même entendu Périclès donner des conseils (455 e 5). Le conseil est par conséquent l'élément qui selon Gorgias, dans la rhétorique, lui permet de triompher de tous, y compris des spécialistes (456 a 1-3), c'est ce qui fait dire, également, à Socrate, de manière ironique, que la rhétorique semble être douée d'une vertu divine. On comprend pourquoi cette confrontation entre rhétorique et philosophie, dont le Gorgias est le théâtre, s'inscrira essentiellement dans le champ délimité par le conseil et le statut du conseiller.

Cette supériorité déclarée du professionnel (dêmiourgos) sur le particulier (idiôtes) n'est pourtant pas toujours la règle chez Platon. Il n'y a pas là inconséquence de sa part. Il convient, pour lever l'ambiguité, d'avoir à l'esprit une autre opposition, celle qui existe entre public et privé. De fait, celui qui revendique le statut de "professionnel" et même de professionnel du conseil, c'est en effet l'homme politique, le rhéteur, le sophiste. Il suffit en ce sens de faire référence à une notion sophistique - même si Platon et Aristote la reprennent par ailleurs pour leur propre compte, j’y reviendrai: la notion d'euboulia, de "bon conseil". L'euboulia, en effet, qui fait l'objet de l'enseignement de Protagoras, porte sur les affaires de la maison et les affaires de la cité et, concernant ces dernières, permet d'être le plus à même de les conduire par l'action et par la parole (prattein kai legein) (Protagoras, 319 a 2). En ce sens, le sophiste et le rhéteur sont bien des professionnels de la politique et opèrent dans la sphère publique.

${ }^{5}$ Ce partage que Socrate opère entre le conseil donné par le spécialiste et le conseil donné par celui qui, précisément, n"y connaît rien n'est pas seulement présent dans le Gorgias. On le retrouve, notamment, dans le Premier Alcibiade (106 d-107 c), dans le Protagoras (319 c 1-7; $319 \mathrm{~d} 6$ ), dans le Théétète (172 a 7). La critique socratique du conseil qui n'est pas donné par le spécialiste, par le professionnel, fait partie intégrante de sa critique des rhéteurs, des sophistes mais aussi, comme c'est le cas dans le Gorgias, des hommes politiques eux-mêmes, comme Thémistocle ou Périclès (voir le Gorgias 519 c 2-3). 
Voilà pourquoi certains textes de Platon soutiennent que le particulier, parfois, est plus apte que le professionnel à donner des conseils. C'est le cas dans le Politique (259 a 1-4), dialogue dans lequel Platon se met en quête de l'authentique politique: "Supposons que quelqu'un qui est un simple particulier (idiôteuôn), soit capable de donner des conseils (sumbouleuein) à un médecin public (dêmosieuontôn iatrôn), n'est-il pas nécessaire de lui donner le nom qui est celui-là même de l'art exercé par celui à qui il prodigue ses conseils?" La question pourrait sembler relever de la perspective des rhéteurs qui précisément prétendent être plus aptes à donner des conseils que les architectes, les constructeurs de navires, voire les médecins, comme c'est le cas pour Gorgias (Gorgias, 456 a sqq.), il ne s'agit pourtant pas de cela. La suite du Politique en témoigne: "Ne dirons-nous pas de quiconque habile à conseiller (parainein) un homme régnant sur des contrées, même s'il est lui-même un simple particulier (idiôtês), qu'il possède le savoir (epistêmên) que le dirigeant devrait lui-même avoir acquise?" (259 a 6-8). Par une forme de renversement, celui qui mérite le titre de professionnel: médecin, roi, ce n'est plus l'homme public, mais le simple particulier, l'homme privé, à la condition qu'il soit capable de donner des conseils à l'homme public, capacité de donner des conseils qui n'est que le signe de la principale qualité, pour Platon, de l'authentique conseiller: le savoir (epistêmê). C'est à cette unique condition que le particulier pourra prétendre donner des conseils à l'homme public. Si Gorgias affirme être supérieur au médecin, c'est en vertu du pouvoir de son logos qui persuade en flattant; si le simple particulier peut l'être, c'est en vertu, selon Platon, de la détention d'un savoir.

C'est également en ce sens que l'opposition professionnel/non professionnel (dêmiourgos/idiôtês) recouvre l'opposition public/privé (dêmosion/idion). De fait, dans l'Apologie de Socrate (31 c 4-7) Socrate affirme: "Peut-être semblera-t-il étrange, qu'alors que moi je distribue en privé (idia) mes conseils (sumbouleuô) à droite et à gauche en me mêlant de beaucoup de choses, je ne me risque pas à m'occuper des affaires publiques (dêmosia) et à monter à la tribune de l'Assemblée du peuple pour donner des conseils (sumbouleuein) à la cité sur ce qui vous concerne" (31 c 4-7). C'est bien ce que Calliclès reproche à Socrate dans le Gorgias, précisément de s'adonner à cette occupation éminemment privée qu'est la philosophie: 
“[...] Car voilà ce qu'autrefois je disais, il appartient à cet homme-là, fût-il doué d'un excellent naturel, de finir par ne pas se comporter de manière virile (anandrô), lorsqu'il fuit le centre de la cité (ta mesa tês poleôs) et ces places sur lesquelles, comme le dit le poète, se font remarquer les hommes, passant le reste de sa vie, enfoncé dans un coin, à chuchoter avec trois ou quatre adolescents, sans jamais rien proférer de libre, de grand ou du moins de convenable.".

\section{Epistêmê, eunoia, parrhêsia}

Les développements platoniciens sur la notion de conseil se fondent par conséquent sur cette opposition entre le conseil rhétorique, d'une part, élément-clé, en raison de sa possible appartenance exclusive à la sphère du discours, de la pratique des rhéteurs et des sophistes et le conseil au sens philosophique, d'autre part, qui se distingue du précédent car il met en œuvre un discours qui se fonde sur un savoir. L'intérêt du passage que je souhaiterais tout particulièrement aborder réside néanmoins avant tout dans le fait qu'il introduit, aux côtés du savoir, deux autres vertus de l'âme du conseiller: bienveillance et franc-parler qui permettent de compléter et de systématiser la conception platonicienne du conseil, et plus encore du conseiller, du sumboulos, telle qu'elle apparaît également dans de nombreux dialogues de Platon ainsi que dans la Lettre VII. Le but sera notamment de montrer que l'authentique conseil est indissociable, pour Platon, de la personne du conseiller, qui présente, comme le suggère le passage, des qualités d'âme. Le texte central auquel je me réfère est le suivant:

Je pense en effet que, pour soumettre comme il faut une âme à l'épreuve pour déterminer si elle mène ou non une vie correcte, il faut donc avoir trois qualités que toi tu possèdes toutes: le savoir (epistêmên), la bienveillance (eunoian) ${ }^{7}$ et le franc-parler (parrhêsian). Je rencontre en effet bien des gens qui ne sont pas capables de me mettre à l'épreuve car ils ne sont pas savants (mê sophoi) comme toi. Il y en a d'autres qui sont savants, mais qui ne veulent pas me dire (legein) la vérité (alêtheian) parce qu'ils ne se préoccupent pas (mê

\footnotetext{
${ }^{6}$ Platon, Gorgias, 485 d 3-e 2.

${ }^{7}$ Certains manuscrits notent doxan et non eunoian. Voir sur ce point le commentaire de E. R. Dodds, dans son édition du Gorgias, ouvr. cit. p. 281 en regard de 487 a 3.
} 
kêdesthai) de moi comme tu le fais. En ce qui concerne ces étrangers - Gorgias et Pôlos - ils sont savants et [b] de mes amis (philô), ils manquent par trop de franc-parler et ont plus de honte qu'on n'en doit avoir. Comment, en effet, ne serait-ce pas le cas? Ils en sont venus à un tel degré de honte (aiskhunês) que la honte leur a donné l'audace à l'un et à l'autre de se contredire, devant une nombreuse assistance, et cela à propos des sujets les plus importants. Toi tu possèdes tous ce que les autres n'ont pas. Tu es éduqué (pepaideusai) comme il faut, comme l'attestent beaucoup de gens à Athènes, et tu es bienveillant à mon égard. [c] La preuve (tekmêriô) que j’en ai? Je vais te le dire. Je sais Calliclès, que, à vous quatre, vous avez formé une association pour l'étude de la sagesse (sophias): toi, Tissandre d'Aphidna, Andron, fils d'Androtion et Nausicyde de Colarge, et je vous ai entendus un jour délibérer (bouleuomenôn) sur la question de savoir jusqu'où il fallait s'exercer à la sagesse. L'opinion qui prévalut parmi vous, je le sais, fut celle selon laquelle il ne fallait pas trop prendre à cœur de philosopher (philosophein) en toute rigueur, [d] vous vous exhortiez (parekeleuesthe) les uns les autres à prendre garde de ne pas, en devenant plus sages (sophôteroı) qu'il ne faut, finir par vous corrompre à votre insu. Ainsi, quand je t'entends me donner les mêmes conseils (sumbouleuontos) qu'à tes plus proches compagnons (hetairotatois), c'est une preuve suffisante à mes yeux que tu es, à mon égard, véritablement bienveillant. Enfin, le fait que tu parles franchement (parrhêsiazdesthai) et sans honte (aiskhunesthar); c'est ce que toi-même tu affirmes et le discours que tu as tenu il $\mathrm{y}$ a peu s'accorde avec ton affirmation ${ }^{8}$.

La pierre de touche dont il est question dans ce texte sert à tester les âmes et non les activités. De manière plus large, l'ensemble du Gorgias se donne pour but de caractériser les hommes bien plus que leurs pratiques, comme en témoigne, au début du dialogue, cette volonté de Socrate de s'interroger sur le rhéteur plus encore que sur la rhétorique (449 a 1-6) $)^{9}$. C’est donc bien le rhéteurconseiller qu'il s'agit de caractériser et non la rhétorique elle-même, si ce n'est que secondairement; c'est donc bien aussi peut-être le philosophe, authentique conseiller, qu'il conviendra, par contraste, de caractériser.

\footnotetext{
8 Platon, Gorgias, 486 e 6-487 d 7.

9 Voir FRIEDLÄNDER, P. Gorgias. In: ERICKSON, K. V. Plato: True and Sophistic Rhetoric. Amsterdam: Rodopi, pp. 91-127, 1979, particulièrement p. 92: « The persons of the drama confront each other, directly expressing themselves and their objective differences » (reprise de FRIEDLÄNDER, P. Plato 2, The Dialogues. First Period. trad. de l'allemand par H. Meyerhoff, New York: Bollingen Series LIX, Pantheon Books, 1964, pp. 244-272 (p. 245).
} 
L'exigence, pour le conseiller, tel que l'entend Platon, de posséder le savoir: epistêmê, ne fait que reprendre cette distinction, ménagée par Socrate au début du Gorgias, à laquelle j’ai fait référence précédemment, entre rhétorique de persuasion de croyance et rhétorique de persuasion d'enseignement ou de science ${ }^{10}$. Il est en outre possible de faire référence au texte du Politique, également précédemment cité, dans lequel l'authentique politique sera, en définitive, non celui qui exerce une fonction publique, mais cet obscur idiôtês qui possède le savoir politique. C'est vraisemblablement pour cette raison que Socrate, déjà, dans le Gorgias, affirme que le seul qui, véritablement, à Athènes, pratique la politique et s'occupe des affaires de la cité, c'est lui-même $(521 \mathrm{~d} \mathrm{6-8})^{11}$. On pense également à ce passage du Phèdre ( $260 \mathrm{~d}$ 5-7), si important pour la notion de conseil dans la mesure où il introduit la notion de psychagogia, où l'art rhétorique se met lui-même à parler et à donner des conseils: "Moi, en effet, je ne force personne (ouden [...] anagkazdô) à apprendre à parler (manthanein legein), quand il ignore la vérité (agnoounta talêthes); mais mon conseil (sumboulê), si on veut bien le suivre, est de ne me prendre en main qu'après avoir acquis cette connaissance". La possession du savoir, en ce sens, doit précéder la mise en œuvre effective, la "prise en main" de l'art rhétorique authentique.

Il est possible, pour la préciser, de mettre en perspective cette référence au savoir, comme qualité de l'âme du conseiller, dans le Gorgias, avec ce qualificatif de l'âme de celui qui exerce la persuasion de croyance, à savoir, le fait d'être habile à conjecturer (stokhastikê) que Socrate emploie dans le passage suivant:

"Mon opinion est, Gorgias, que [la rhétorique] est une pratique (epitêdeuma) qui n'a rien d'un art (tekhnikon) mais qui est le propre d'une âme habile à conjecturer (stokhastikês), virile (andreias) et naturellement redoutable dans les rapports

\footnotetext{
${ }^{10}$ Sur ce passage, voir notamment COULTER, J. A. The Relation of the Apology of Socrates to Gorgias Defense of Palamedes and Plato's Critique of Gorgianic Rhetoric. In: ERICKSON, K. V. Plato: True and Sophistic Rhetoric, ouvr. cit., pp. 31-69 particulièrement pp. 52-57 (réimpr. de l'article initialement publié dans les Harvard Studies in Classical Philology, 68, pp. 269-303, 1964).

${ }^{11}$ Sur cette affirmation socratique évoquée supra n. 4, sa dimension paradoxale, sur le rapport qu'elle entretient avec d'autres témoignages sur Socrate (Xénophon, Mémorables, 3.9.10) et d'autres dialogues platoniciens, notamment le Charmide (171 d-172a), l'Euthydème (288 e-291 d), la République V (473 c-e) et le Politique, sur le rapport entre philosophie et expertise politique qu'elle implique et, enfin, d'une manière plus générale, sur la place du savoir en politique selon Platon, voir SCHOFIELD, M. Plato Political Philosophy, ouvr. cit., chapitre 4.
} 
humains (phusei deinês prosomilein tois anthrôpois). Cette pratique, je l'appelle, en un mot, flatterie (kolakeian) ${ }^{12}$."

Ce qualificatif: "habile à conjecturer" a de manière certaine une dimension négative, au même titre d'ailleurs que les deux autres qualificatifs employés: "virile" et "redoutable dans les rapports humains", tout simplement parce que ces trois qualificatifs visent précisément à caractériser aux yeux de Socrate l'âme du rhéteur telle que l'entend Gorgias, celui qui, par conséquent, ne pratique qu'une rhétorique de persuasion de croyance, à laquelle justement, parce qu'elle n'est pas science, Socrate refuse le statut de technique. Les différentes occurrences du terme de conjecture et de ses dérivés vont d'ailleurs également dans le sens d'une conception strictement négative de la conjecture. Ainsi en 464 c 6, l'art de flatter s'aperçoit de l'existence des soins du corps et des soins de l'âme, non en vertu d'une connaissance (ou gnousa) mais au moyen d'une conjecture (stokhasamenê); et en 465 a 1-2, la rhétorique est qualifiée de laide par Socrate car "c'est l'agréable qu'elle conjecture (stokhazdetai) et non le meilleur”. En ce sens, son objet même, l'agréable, condamne la rhétorique à ne pas pouvoir accéder à une connaissance scientifique, la cantonne par conséquent à n'être que conjecture et exclut pour elle la possibilité d'accéder au statut de tekhnê. Je renvoie sur ce point au Philèbe 27 e sqq. où le plaisir échappant à toute forme de détermination, ne peut en aucun cas être objet de science ${ }^{13}$. C'est en ce sens également que la rhétorique de Gorgias n'est qu'un savoir-faire (empeiria) ou une routine (tribê). Enfin, au tout début du Lachès, l'habileté à conjecturer contraste, de manière extrêmement significative, non plus avec le savoir, mais avec la libre-parole, lorsque Lysimaque oppose "ceux qui tentent de deviner ce que pense celui qui leur demandent conseil (stokhazdomenoi tou sumbouleuomenou)" à ceux qui pensent qu'il convient de "parler librement (parrhêsiazdesthai)"14.

Epistêmê est certes la qualité nécessaire pour que le conseiller soit authentique conseiller, mais elle n'est pas une qualité suffisante et ne permet pas, à elle seule, de caractériser le conseil. Les deux autres qualités que sont

\footnotetext{
12 Platon, Gorgias, 463 a 6-463 b 1.

13 Cf. sur ce point l'article de ROOCHNIK, D. Socrates Rhetorical Attack on Rhetoric. In: GONZALEZ, F. J. The Third Way. New Directions in Platonic Studies. Boston-London: Rowman \& Littlefield Publishers, pp. 81-94, 1995, particulièrement p. 86.

14 Platon, Lachès, 178 a 1-b 3 . Ce rapprochement est proposé par DODDS, E. R. Platon. Gorgias, ouvr. cit., commentaire de 463 a 6-b 1, p. 225.
} 
eunoia et parrhêsia revêtent un dimension plus décisivement rhétorique dans la mesure où elles caractérisent un état que l'âme du conseiller possède vis-à-vis de son interlocuteur. Un tel état de fait conduit d'emblée à la considération suivante, ce qui différencie rhétorique de persuasion de croyance et rhétorique de persuasion d'enseignement, c'est bien en effet leur dimension, ou non, scientifique; en revanche, la convocation de ces qualités que sont eunoia et parrhêsia, contribue à mettre l'accent, de manière décisive, non pas sur la connaissance que celui qui parle a de son objet, mais sur l'intention qui est la sienne vis-à-vis de celui à qui il s'adresse - Aristote, en ce sens, sera parfaitement fidèle à cette inspiration de Platon lorsqu'il affirmera, dans plusieurs de ses traités ${ }^{15}$ que ce qui compte, ce n'est pas la capacité mais l'intention. D’où, dans le Gorgias, la mention d'une qualité qui, à la différence du savoir, contribue à inscrire le logos dans sa dimension relationnelle, qualité emblématique également de l'intention à l'égard de son interlocuteur dont celui qui parle anime son logos: la bienveillance. Une bienveillance dépourvue de savoir cependant serait impuissante, l'intention, en ce sens, ne suffit pas, ce qui justifie pourquoi la bienveillance est placée en deuxième position.

Si Socrate affirme que Calliclès a de la bienveillance à son égard, c'est parce que Calliclès, lui-même, l'a affirmé en 486 a 4 . La référence à l'eunoia est d'ailleurs présentée par Calliclès conjointement à celle d'amitié (485 e 2-3), il s'adresse à Socrate, dit-il, comme on le ferait à un frère (485 e 6). Dans la suite du Gorgias, la notion d'eunoia est à nouveau associée à celle d'amitié, de philia ${ }^{16}$. Ainsi, Gorgias et Pôlos sont-ils qualifiés de bienveillants parce qu'ils sont les amis de Socrate. Socrate affirme qu'il a entendu Calliclès donner à ses amis, à ses plus proches compagnons (hetairotator), les mêmes conseils qu'à lui-même, ce qui l'a conduit à en déduire que Calliclès était bienveillant à son égard ${ }^{17}$. De fait, la bienveillance est très souvent associée à l'amitié, comme c'est le cas dans le Phèdre (241 c 7-8) et surtout 255 b 3-7 où elle apparaît même comme la marque de l'authentique amitié, précisément car elle n'a souci que de l'intérêt de l'autre. C'est en ce sens que dans le Gorgias (487 a 6), la bienveillance correspond au fait de "prendre soin" de l'autre, de s'en préoccuper, comme en témoigne l'usage de kêdesthai.

15 Aristote, Métaphysique, 1004 b 22-26; Éthique à Nicomaque, 1127 b 14; Réfutations Sophistiques, 165 a 30-31; Rhétorique, I, 1, 1355 b 17-21.

${ }_{16}$ Platon, Gorgias, 487 b 1, et 487 d 3 passage cité supra pp. 68, n. 8.

${ }_{17}$ Voir MCKIM, R. Shame and Truth in Plato's Gorgias, art. cit., p. 40. 
Il est permis de douter, bien entendu, que Calliclès soit authentiquement bienveillant à l'égard de Socrate; par la suite, d'ailleurs, dans le dialogue (499 c 2-4), Socrate lui même se prend à douter que Calliclès soit véritablement son $\mathrm{ami}^{18}$. Notons néanmoins que l'authentique bienveillance n'exclut pas la critique ou la correction, c'est d'ailleurs en ce sens que Calliclès peut au moins "prétendre" être bienveillant vis-à-vis de Socrate. De manière sans doute plus significative, on peut faire référence au début du Théétète, où Socrate affirme que c'est par bienveillance qu'il détruit les fausses opinions dans l'âme de ceux qu'il accouche (151 c 8), de même que c'est par bienveillance que l'Étranger d'Élée reprend Socrate le Jeune car il a commis une erreur dans sa manière de diviser (Politique, 262 c 3). On mesure en ce sens tout ce qui sépare le conseil, dans sa dimension bienveillante, d'une rhétorique conçue comme un sport de combat, dotée essentiellement d'une dimension éristique, ou d'une rhétorique qui utilise les armes de la flatterie pour triompher. On mesure aussi, par contraste, tout ce qui le rapproche de la maïeutique et de la dialectique, comme le suggèrent les deux textes du Théétète et du Politique où apparaît la notion d'eunoia, le but n'étant pas de faire chuter son interlocuteur, mais au contraire de le faire progresser. L'intention, une fois encore prévaut, la réfutation peut être sophistique, elle peut être socratique; la différence entre l'une et l'autre ne réside pas dans la puissance mais dans l'intention.

Il en résulte également que cette place qu'occupe l'eunoia dans le conseil authentique contribue à faire de lui une pratique essentiellement privée. Si Socrate se contente de chuchoter dans les coins avec quelques adolescents ${ }^{19}$, c'est parce que le conseil bienveillant implique la mise en œuvre d'une relation personnelle et actuelle avec l'interlocuteur; il ne peut s'agir d'un conseil stéréotypé qui s'adresserait d'une manière indifférenciée à une foule. Surtout, ce n'est pas un logos qui vise à prendre le dessus sur l'autre et qui œuvre uniquement pour l'intérêt propre de celui qui le prononce. En cela, contrairement au rhéteur de profession "redoutable dans les rapports humains" 20 , Socrate est devenu, à cause précisément de la philosophie, de sa vocation bienveillante, de son cantonnement dans la sphère privée, "inexpérimenté" en tout ce qui concerne les rapports humains,

18 Le thème de l'amitié est très présent au début de l'échange entre Socrate et Calliclès (482 a 5 ; 485 e 3 ).

19 Platon, Gorgias, 485 d 7-e 1, passage cité supra p. 67, n. 6.

20 Platon, Gorgias, 463 a 8, passage cité supra p. 70, n. 12. 
la vie de la cité21. C'est, en définitive, sa bienveillance qui a conduit Socrate à sa perte, incapable qu'il était de tenir un discours en vue de son propre intérêt, comme le souligne Calliclès dans le passage suivant:

'Voilà ce qu'il en est selon la vérité. Or c'est ce que tu reconnaîtras si tu t'orientes vers de plus hauts objets et renonces désormais à la philosophie. La philosophie, certes, a son charme, Socrate, à condition qu'on s'y applique avec modération dans la jeunesse; mais, si l'on y consacre plus de temps qu'il ne faut, cela est ruineux pour tout être humain. Supposons en effet que, fût-il doué d'un excellent naturel, il se soit consacré à la philosophie au-delà de la jeunesse, il est nécessaire qu'il devienne inexpérimenté (apeiron) [d] concernant tout ce en quoi il faut être expérimenté (empeiron) pour devenir un homme accompli (kalon kagathon) et estimé (eudokimon). En effet, il devient inexpérimenté concernant les lois qui sont celles de la cité; concernant les discours (tôn logôn) qu'il faut utiliser dans les contrats, qu'ils soient privés (idia) ou publics (dêmosia), qui sont passés dans le cadre des rapports humains (homilein tois anthrôpois); concernant les plaisirs (hêdonôn) et les désirs (epithumiôn) des hommes (anthrôpeiôn). En un mot, il devient, de manière générale, inexpérimenté en ce qui concerne les mœurs (tôn êthôn) 22 ."

C’est en ce sens, également, que Calliclès accuse Socrate de manquer de virilitée ${ }^{3}$, par contraste, précisément avec celui qui pratique la rhétorique et qui se caractérise par son courage ${ }^{24}$. Le Gorgias, on le sait, est placé sous l'égide de la guerre et du combat, qui constituent d'ailleurs les deux premiers mots du dialogue. En ce sens, le courage dont il est question ici est bien celui

\footnotetext{
${ }^{21}$ En ce sens, on peut faire référence à l'article de ROCCO, C. Liberating Discourse: The Politics of Truth in Plato's Gorgias. Interpretation, 23, pp. 361-385, 1996, particulièrement pp. 373-374: « To claim a monopoly on the true art of politics [l'auteur fait allusion à l'affirmation de Socrate selon laquelle, en $521 \mathrm{~d} 6-8$, il est le seul de tous les Athéniens à authentiquement pratiquer l'art politique] and then refuse to participate in the "official" discourse of the city (or do so incompetently) indicates either the failure of philosophy as a political ideal or else the failure of the Athenian citizens to respond to Socratic philosophical education. In both cases, Socratic dialogue remains marginalized, performing its work at the interstices between public and private, trespassing on both, at home in neither ».

22 Platon, Gorgias, 484 c 4-484 d 7.

${ }^{23}$ Platon, Gorgias, 485 d 4, passage cité supra p. 67, n. 6.

${ }^{24}$ Platon, Gorgias, 463 a 7, passage cité supra p. 70, n. 12.
} 
du combattant, de celui qui considère la rhétorique comme une arme. Par une forme de renversement, cependant, Socrate montre en 518 c sqq. que le courage est plutôt le fait de ceux qui donnent des conseils à la cité afin qu'elle guérisse de ses maux que de ceux qui l'ont gavée et l'ont flattée par leurs actes et par leurs discours. On peut dire, en ce sens, que, dans le Gorgias, le courage change de camp ${ }^{25}$, n'est plus l'apanage du rhéteur précisément parce qu'il est un flatteur - mais du philosophe. On trouve une perspective similaire dans la Lettre VII qui témoigne sans doute de l'expérience de conseiller politique de Platon auprès de Denys de Syracuse. Dans ce passage, où médecine et politique sont une fois encore présentées dans un rapport analogique, la flatterie est explicitement associée à la lâcheté. En effet, celui qui accepterait de se mettre au service d'un mauvais régime, qui accepterait de ne donner des conseils que sur la manière dont les désirs de ceux qu'ils conseillent seront susceptibles de se réaliser aisément et rapidement, "celui qui se résignerait à donner ce type de conseil, affirme Platon, je considérerais qu'il n'est pas un homme (anandron), celui qui ne s'y résignerait pas, je considérerais qu'il est un homme" (331 a 4-5). Platon, en renonçant à conseiller Denys a finalement fait preuve de courage, courage qui réside non pas dans le fait de parler, mais au contraire, de se taire ${ }^{26}$.

On mesure alors la portée de la référence à la parrhêsia comme qualité du conseiller et les rapports qu'elle entretient avec l'authentique courage. La parrhêsia est un des termes clés de la démocratie athénienne ${ }^{27}$. Alors que l'iségoria donne le droit à chacun de prendre la parole à l'Assemblée, la parrhêsia valorise le parler vrai, le fait de tout dire, indépendamment de toute forme de précaution rhétorique ${ }^{28}$, avec éventuellement tous les risques que

\footnotetext{
25 Sur ce point, voir notamment l'analyse de BUZZETTI, E. The Injustice of Callicles and the Limits of Socrates's Ability to Educate a Young Politician. Ancient Philosophy, 25, pp. 25-63, 2005, plus particulièrement, p. 44: «This fearfulness helps explain, moreover, why Callicles is not receptive to Socrates in the dialogue: he is unable to free himself from the ascendancy that the demos has over his mind. He would like to escape the enslaving influence of the demos's praises and blames, yet he has made himself one of the demos, praising and blaming whatever the demos praises and blames; he is contemptuous of the demos's lowliness, yet he craves its friendship because he is afraid of its power (481 d 5-482 a 2, 483 b 4-484 c 3) ».

${ }^{26}$ Voir également Platon, République, VIII, 567 b 3-10 et la nécessité pour le tyran de se débarrasser de ceux qui sont assez courageux (andrikôtatoi ontes) pour parler franchement (parrhêsiazdesthai), référence proposée par VAN RAALTE, M. Socratic Parrhesia and its Afterlife in Plato's Laws, ouvr. cit., p. 290, n. 34.

${ }^{27}$ A cet égard, cf. Platon, République, VIII, 557 b 5.

${ }^{28}$ Sur la notion de parrhêsia chez Platon, on pourra se reporter à FOUCAULT, M. Le gouvernement de soi et des autres. Cours au Collège de France (1982-1983). Édition établie, sous la direction de
} 
cela peut comporter pour celui qui adopte ce franc-parler. Dans le texte du Gorgias, la parrhêsia entre en opposition avec la honte (aiskhunê), ce sentiment de honte - ou de réserve - qui aurait conduit Gorgias et Pôlos à se contredire en public. Le propos est vraisemblablement, là aussi, ironique ${ }^{29}$. On comprend néanmoins dans quelle mesure la parrhêsia est partie intégrante du conseil authentique et est indissociable du savoir et de la bienveillance: du savoir, parce que parler librement alors qu'on n'a que des opinions susceptibles d'être fausses ne peut en aucun cas constituer le bon conseil, de la bienveillance, car, comme le suggère le passage, en dépit de sa dimension ironique, on peut être savant et bienveillant, mais ne pas oser dire la vérité, et, par conséquent, ne pas donner le meilleur conseil.

De quelle nature est cette honte, cette réserve, qui conduit à ne pas parler franchement? Platon cite délibérement l'eunoia avant la parrhêsia. La raison en est qu'il s'agit de préciser les qualités du conseiller, or, la bienveillance est la marque même du conseil, en tant qu'il est donné, dans son intérêt, à l'ami, au compagnon; cette amitié, pourtant, peut mal s'accommoder de la vérité qui peut blesser l'ami et rompre par conséquent l'amitié. Ne voulant pas blesser ses amis, connaissant la vérité, on ne la leur dit pas, non pas par désintérêt, mais par honte, comme c'est le cas de Gorgias et de Pôlos, qui "amis" (c'est le terme que Socrate utilise) de Socrate n'osent lui parler franchement. Pourtant, il convient d'avoir à l'esprit la différence entre amitié et bienveillance, telle notamment qu'elle est précisée dans le Phèdre. La bienveillance vise à l'intérêt de l'autre, c'est une amitié totalement

EWALD, F., FONTANA, A., par GROS, F. Paris: Gallimard-Le Seuil, 2008; MONOSON, S. S. Plato's Democratic Entanglements: Athenian Politics and the Practice of Philosophy. Princeton: Princeton University Press, 2000, pp. 162 sqq.; JEANMART, G. Généalogie de la docilité: dans l'Antiquité et le Haut Moyen Âge. Paris: Vrin, 2007, pp. 17-57; MARKOVITS, E. The Politics of Sincerity: Plato, Frank Speech and Democratic Judgment. Penn State Press, 2008, pp. 92 sqq. On pourra se reporter en outre à l'ouvrage de SAXONHOUSE, A. W. Free Speech and Democracy in Ancient Athens. Cambridge: Cambridge University Press, 2006, qui mène une analyse conjointe des notions de parrhêsia et d'aidôs dans la démocratie athénienne.

${ }^{29}$ Cf. 487 b1-3; Sur cet aspect du dialogue et la notion problématique de « honte » dans le Gorgias, voir BENSEN CAIN, R. Shame and Ambiguity in Plato's Gorgias. Philosophy and Rhetoric, 41, pp. 212-237, 2008. Voir également la thèse de MCKIM, R. Shame and Truth in Plato's Gorgias, qui prend le contrepied de la conception que Dodds développe dans son commentaire en regard de 482 c 4-483 c 6 in Plato. Gorgias, ouvr. cit., p. 263. Selon McKim, en effet, "Socrates' implicit view of the relationship between shame and belief is the exact opposite of Callicles'. Whereas shame is for Callicles an unnatural feeling that inhibits our real preference for vice, Socrates believes to the contrary, on my reading, that our shame about vice is a natural sign that deep down we really prefer virtue. », art. cit., p. 39). II prend appui sur cette thèse afin de démontrer le caractère hautement ironique de l'affirmation socratique selon laquelle l'âme de Calliclès est douée de parrhêsia (p. 40). 
désintéressée - voir sur ce point les développements d'Aristote sur le bienveillant, dans l'Éthique à Nicomaque (VIII, 2, 1155 b 32-34), décrit comme celui qui veut du bien à un autre, même quand cela n'est pas réciproque. En ce sens, la parrhêsia complète l'eunoia, elle fait qu'elle est authentique eunoia et non amitié qui recherche la réciprocité. La parrhêsia est la qualité qui permet, en définitive, d'accorder vérité et amitié, de faire en sorte que la première prévale entre amis, comme marque même de l'amitié en tant qu'elle vise le bien de l'ami avant tout, y compris au prix de l'amitié, et qu'elle est donc, par conséquent, bienveillance.

C'est peut-être en ce sens que la parrhêsia conduit parfois à l'incivilité, comme le souligne Socrate dans le Gorgias (462 e 6): "Peut-être est-ce par trop incivil, rustre (agroikoteron) de dire la vérité (to alêthes eipein)". En ce sens, la parrhêsia, loin de rendre redoutable dans les rapports humains, rejette celui qui la pratique en marge de la société des hommes, ou du moins de ces relations conventionnelles que les hommes entretiennent entre eux et dont il est question dans le Gorgias, en $484 \mathrm{~d} 4$. On comprend enfin pourquoi la parrhêsia, au même titre d'ailleurs que l'eunoia, sont l'une et l'autre incompatibles avec le fait même de monnayer ses conseils, comme le déclare Socrate: "concernant cette activité qui s'emploie à déterminer de quelle façon on deviendra le meilleur possible et on administrera de la meilleure façon sa maison ou la cité, il est d'usage de tenir pour une chose laide, semble-t-il, de ne pas donner de conseil (mê sumbouleuein), s'il n'y a pas quelqu'un pour donner de l'argent" (Gorgias 520 e 2-5). Monnayer ses conseils, comme le font les sophistes et les rhéteurs, consiste à considérer que les seules relations à l'œuvre entre les hommes sont finalement de nature purement contractuelle, condamnant toute forme d'échange "naturel" et "spontané", animé par la bienveillance et usant de libre-parole, à être reléguée au rang de pratique honteuse et clandestine, comme le suggère, encore une fois, Calliclès en $485 \mathrm{~d}$ 3-e 2.

La notion de parrhêsia est présente dans de nombreux dialogues de Platon, je voudrais simplement faire une référence à un passage qui est significatif, me semble-t-il, de la dimension avant tout conseillère de la parrhêsia et qui éclaire en ce sens le texte du Gorgias. Le Lachès, tout particulièrement, associe le logos authentiquement conseiller à la parrhêsia et au courage. Lachès évoque le caractère ambigu de son rapport au discours: philologie, mais aussi misologie. Ce qu'il aime, dit-il, c'est écouter dialoguer à propos de la vertu un homme vraiment homme, un homme digne des 
discours qu'il tient. Cet homme-là, selon lui, est un musicien, qui parvient à atteindre l'harmonie entre ses actes et ses paroles, l'harmonie avec lui-même, harmonie que l'on trouve dans une musique qui inspire le courage. Ce qu'il hait, au contraire, ce sont les discours qui ne ménagent pas cet accord entre les actes et les paroles, y compris s'ils sont le fait de bons orateurs. Les actes mêmes de Socrate font qu'il est lui-même digne de bien parler, en toute franchise (pasê parrhêsia), c'est pourquoi Lachès se réjouit d'être conseillé par lui (188 c 4-189 a 5). Cet accord entre les paroles et les actes trouve son expression à la fin du Gorgias et montre dans quelle mesure la libre-parole est associée au courage. C'est Socrate en effet qui fait preuve de parrhêsia et de courage et qui en assume les risques lorsqu'il parle librement; exhortant Calliclès à continuer à lui dire la vérité et à continuer à lui parler librement, ce dernier en vient à lui conseiller, paradoxalement, de se mettre au service des Athéniens, comme le ferait un esclave, et de les flatter (521 a 2-8), en d'autres termes d'abandonner toute forme de parrhêsia, afin d'éviter d'être accusé et condamné.

\section{Les qualités du conseiller dans la Rhétorique et l'Éthique à Nicomaque d'Aristote}

Je voudrais, pour finir, évoquer un texte de la Rhétorique dans lequel Aristote précise les qualités du conseiller, de celui qui pratique le genre "délibératif" (sumbouleutikon genos). Comme on le sait, le genre "conseiller" ou délibératif est celui qui s'exerce de manière privilégiée à l'Assemblée du peuple et, de manière plus générale, dans le domaine politique. La parenté flagrante entre le début du passage du Gorgias (486 e 6-487 b 5) qui fait l'objet de cette étude et le passage suivant de la Rhétorique, tant au niveau des qualités évoquées que de la construction, éclaire rétrospectivement le texte de Platon.

Les raisons pour lesquelles ceux qui parlent inspirent eux-mêmes confiance (pistous) sont au nombre de trois car il y a trois motifs pour lesquels nous accordons notre confiance (pisteuomen) en dehors des démonstrations. Ce sont la prudence (phronêsis), l'excellence (aretê) et la bienveillance (eunoia). Les orateurs, en effet, sont déçus dans leurs entreprises lorsqu'ils parlent ou lorsqu'ils donnent des conseils faute soit de toutes ces qualités soit faute d'une seule d'entre elles. Soit, en effet, faute de prudence (aphrosunên) ils n'ont pas des opinions correctes (ouk orthôs doxazdousin), 
soit, bien qu'ayant des opinions correctes, ils ne disent pas leur opinion par méchanceté (mokhthêrian), soit, ils sont prudents (phronimoi) et honnêtes (epieikeis), mais pas bienveillants (eunoi), c'est pourquoi il est possible (endekhetai) qu'il ne donnent pas les meilleurs conseils (mê ta beltista sumbouleuein) alors qu'ils les connaissent ${ }^{30}$.

Les qualités, il est vrai, ne sont pas tout à fait les mêmes, à l'exception de l'eunoia qui est présente dans les deux textes. Aristote substitue la phronêsis à l'epistêmê platonicienne, les deux termes ne reviennent certes pas au même, surtout pour Aristote, notamment dans l'Éthique à Nicomaque. Le terme d'arêtê est suffisamment vague pour qu'on puisse lui substituer le courage ou la parrhêsia, même s'il faut plus justement le considérer avant tout comme vertu morale en général. Ce sur quoi je voudrais insister, c'est sur le fait que les deux passages adoptent une construction analogue qui n'est certainement pas un hasard ${ }^{31}$. Cette analogie est particulièrement significative, non pas parce qu'Aristote veut dire la même chose que Platon, mais plutôt, précisément, parce qu'il ne le veut pas. Alors que les qualités énumérées par Platon relèvent à la fois de l'éthique et de la rhétorique, rhétorique authentique précisément parce qu'elle est éthique; les qualités énumérées par Aristote doivent être avant tout considérées dans leur dimension exclusivement rhétorique. Certes, phronêsis, arêtê, et même eunoia - dans une moindre mesure cependant - occupent une place centrale, comme on le sait, dans les traités éthiques, mais elles ne jouent pas ce rôle dans la Rhétorique. Aristote s'y emploie, en effet, à prendre le contrepied des développements platoniciens sur la rhétorique dans le Gorgias, affirmant notamment que la rhétorique est une tekhnê (I, 1, 1354 a 11), une dunamis (I, 2, 1355 b 25), puissance des contraires qui est, par conséquent, dénuée de toute dimension morale. En cela, il s'inscrit, d'une certaine façon, davantage dans le prolongement des propos de Gorgias dans le Gorgias, que dans le prolongement de la conception que Platon développe, lui-même, par l'intermédiaire de Socrate, dans ce dialogue, voire de celle que Platon met en œuvre, dans le Phèdre, dans sa recherche d'une rhétorique authentique qui serait à la fois connaissance des discours et des âmes.

\footnotetext{
${ }^{30}$ Aristote, Rhétorique, II, 1, 1378 a 6-15. Voir HAUSER, G. A. Aristotle on Epideictic: The Formation of Public Morality. Rhetoric Society Quarterly, 29, p. 5-23, 1999, particulièrement, p. 9 qui soutient la thèse selon laquelle les qualités du rhéteurs sont aussi les qualités du phronimos et la critique qu'en propose MCADON, B. Reconsidering the Intention or Purpose of Aristotle's Rhetoric. Rhetoric Review, 23, p. 226228, 2004.

${ }^{31}$ Voir notamment WOERTHER, F. L'èthos aristotélicien, genèse d'une notion rhétorique. Paris: Vrin, 2007, pp. 217 sqq.
} 
Ainsi, ces traits de l'êthos de l'orateur, de celui qui, à l'Assemblée ou en privé, pratique le genre délibératif, doivent être compris non pas comme étant possédés par l'orateur, mais comme semblant l'être. L'orateur doit par conséquent s'efforcer, par le biais de son logos, de suggérer qu'il est prudent, vertueux et bienveillant, même si, en définitive, il ne l'est pas. Ce n'est donc pas ce qu'est l'orateur, mais ce qu'il semble être - prudent, vertueux, etc. pour celui qui l'écoute, qu'il faut considérer. L'analogie de construction des deux passages prend alors tout son sens. Si l'absence réelle de savoir rend incapable de tester les âmes et par conséquent de leur donner de bon conseil pour Platon, l'apparent défaut de phronêsis chez l'orateur rendra son discours inefficace auprès de l'auditeur, tout simplement parce qu'il n'inspirera pas confiance (pistis). En revanche, si l'orateur parvient à faire croire qu'il est prudent, alors, il convaincra, même si de fait, il n'est pas prudent. La prudence cependant ne suffit pas. Imaginons un prudent méchant, comme nous y invite Aristote dans la Rhétorique, avec toutes les difficultés que cela peut comporter dans une perspective éthique quand on connaît le lien strict qui existe, dans les Éthiques entre les vertus morales et la phronêsis. Mais éthique, précisément, la perspective ne l'est pas dans la Rhétorique, il ne s'agit pas de ce que l'on est, des vertus que l'on possède, mais de ce que l'orateur semble posséder. Si l'orateur peut réussir, par son $\log o s$, à suggérer qu'il est prudent, il peut échouer à suggérer qu'il est vertueux, il peut donc être soupçonné, comme le souligne le texte, de ne pas donner de bons conseils, ou plutôt d'en donner de mauvais, par méchanceté, par traîtrise et par volonté délibérée de nuire. Enfin, l'orateur peut sembler prudent, vertueux - il ne donnera pas délibérement de mauvais conseils -, mais sembler manquer de bienveillance. Ce manque d'intérêt manifeste pour l'auditeur, celui qui reçoit le conseil, conduira ce dernier à penser que l'orateur en question ne donnera pas les meilleurs conseils, non par vice, mais par une forme d'indifférence. Là encore, le conseil ne sera pas un "bon" conseil pour Aristote, non pas parce qu'il ne conseillera pas le meilleur, mais parce qu'il n'atteindra pas le but de la rhétorique: persuader, ou plus précisément discerner dans chaque cas le potentiellement persuasif (I, 2, 1355 b 25-27).

On mesure ici toute la distance qui sépare la conception d'Aristote de celle de Platon, tout particulièrement dans le Gorgias. Le but d'Aristote est simple, contre Platon, mais aussi sans doute pour mieux prendre possession d'un terrain jusque-là occupé par les rhéteurs - comme il le fera d'ailleurs vis- 
à-vis des sophistes avec sa dialectique - Aristote développe une conception strictement et exclusivement rhétorique du conseil, reprenant pour son propre compte, au moins en partie, cette conception de la rhétorique qui vise la pistis, rhétorique que Platon, précisément, refuse, rhétorique qu'Aristote veut, en ce qui le concerne, mettre au service de la politique, sans pour autant que l'une et l'autre se confondent ${ }^{32}$.

Pourtant, si la Rhétorique est bien le seul traité dans lequel Aristote expose sa conception du conseil (sumbouleuein), le livre VI de l'Éthique à Nicomaque propose des développements sur une autre notion, que nous avons déjà évoquée, et qui a trait elle aussi, au conseil. Il s'agit de l'euboulia, bonne délibération ou bon conseil ${ }^{33}$, états caractéristiques de la phronêsis qu'Aristote développe conjointement avec deux autres notions: la compréhension (sunêsis) et la bienveillance ou indulgence. Je voudrais brièvement évoquer trois points. Tout d'abord Aristote refuse à l'euboulia, contre Platon, le statut d'epistêmê (1142 b 1), mais il lui refuse aussi le statut de conjecture, serait-elle bonne conjecture (eustokhia) (1142 b 7). La référence à la définition de l'euboulia comme savoir, par Platon, dans la République (IV, 428 b 6-7), est transparente, mais dans le Gorgias aussi, le savoir est considérée comme la première qualité de celui qui est apte à tester les âmes, à être, par conséquent, authentique conseiller. Pour Aristote, le bon conseil n'est pas savoir car "celui qui conseille recherche et calcule" (Éthique à Nicomaque, VI, 1142 b 2-3), en ce sens, le bon conseil est aussi bonne délibération; mais il ne sera pas non plus conjecture, ni même "bonne conjecture" dans la mesure où la bonne conjecture se passe de raisonnement, alors que le bon conseil doit prendre le temps de la délibération, en ce sens, Aristote rejette aussi la conception d'un conseil qui serait avant tout le fruit d'une forme de perspicacité, comme le fait Platon dans le Gorgias, contre les rhéteurs, mais pas pour les mêmes raisons que Platon, contre les sophistes également, je rappelle l'origine sophistique du terme euboulia, cet art de bien

32 Voir sur ce point Rhétorique, I, 2, 1356 a 25-27: « la rhétorique est comme une sorte de rejeton (paraphues) de la dialectique, ainsi d'ailleurs que de l'étude des caractères, qu'il convient d'appeler politique ».

${ }^{33}$ Cf. GAUTHIER, R. A., JOLIF, J. Y. L'Éthique à Nicomaque, Introduction, traduction et commentaire. Louvain-Paris: Publications Universitaires de Louvain-Éditions Béatrice Nauwelaerts, 4 vol., 19702; réimpr. Louvain-la-Neuve-Paris-Sterling, Virginia: Éditions Peeters, 2002, tome II, deuxième partie, commentaire Livres VI-X, p. 509-519. Sur l'euboulia dans l'Éthique à Nicomaque, voir NARCY, M. Être de bon conseil et savoir écouter (Éthique à Nicomaque, VI, 10-11). In: CHATEAU, J.-Y. La vérité pratique: Aristote, Éthique à Nicomaque, Livre VI, Paris: Vrin, p. 117-135, 1997, dont je reprends en partie ici les analyses. 
conseiller dont se prévalait Protagoras dans le Protagoras.

Deuxième point: les notions de compréhension et d'indulgence sont particulièrement intéressantes dans la mesure où, à l'instar de l'eunoia et de la parrhêsia, elles contribuent à faire que le bon conseil soit bien authentiquement conseil et non pas simple délibération à propos d'une action que l'on s'apprête soi-même à accomplir. En ce sens, le bon conseil, pour Aristote, est délibération effectuée en vue de l'accomplissement de l'action par un autre que soi. Ce qui fait de l'euboulia un véritable conseil, au plein sens du terme, c'est son association, par Aristote, à la sunêsis. La sunêsis en effet, c'est cette capacité à écouter, quand "c'est un autre qui parle" (1143 a 16), une capacité par conséquent à prendre en compte l'autre, qui vient demander conseil, dans ce qu'il a de plus particulier.

Troisième point et dernier point: l'indulgence dont il est question renvoie également à une capacité tournée vers l'autre, c'est en ce sens que "le prudent (phronimos) est un homme compréhensif (sunetos), bienveillant ou indulgent (eugnômôn ê suggnômôn), car les actes honnêtes sont communs à tous les hommes bons dans leurs relations avec les autres" (1143 a 30-33). On mesure la communauté d'inspiration entre la bienveillance platonicienne et l'indulgence aristotélicienne.

\section{Conclusion}

La conception aristotélicienne du conseil se distingue de celle que Platon met en œuvre, tout particulièrement dans le Gorgias, en ce qu'Aristote s'attache à ménager une place à une conception strictement rhétorique du sumbouleuein - terme qu'Aristote réserve de manière exclusive au sens rhétorique du conseil - dont le but est la persuasion, art du conseil qui exclut en ce sens toute dimension éthique. D’une certaine façon, c'est le conseil rhétorique tel que l'entendent Gorgias ou les rhéteurs de son époque qu'Aristote élève au niveau d'une authentique technique, considérant les qualités du conseiller non plus dans la visée éthique qui était celle du Gorgias, mais dans une visée purement rhétorique. Dans l'Éthique à Nicomaque, Aristote développe une conception authentiquement éthique du conseil, qui dégage également trois qualités du conseiller. C'est alors plus particulièrement la conception du conseil comme science qu'Aristote remet en cause, mettant ainsi l'accent, beaucoup plus que Platon, sur sa dimension délibérante, sur son inscription dans la sphère pratique et sur le fait qu'il 
porte sur les affaires humaines, changeantes et particulières, par définition. Il reste néanmoins que le texte du Gorgias, précisément parce qu'il accorde une place à ces qualités que sont l'eunoia et la parrhêsia, inaugure sans doute une réflexion systématique et organisée sur la notion de conseil, fidèle en grande partie à l'inspiration socratique, à savoir une relation personnelle spécifique entre deux âmes - elles-mêmes dotées de qualités bien spécifiques - par le biais du logos. Les développements platoniciens du Phèdre sur la nécessaire adaptation du discours à l'âme à laquelle il s'adresse dans le cadre de la psychagogia trouvent peut-être leurs premiers linéaments dans ce passage du Gorgias, passage qui constitue également un point de départ privilégié pour la réflexion aristotélicienne sur le conseil. 


\section{Referências bibliográficas}

BENSEN CAIN, R. Shame and Ambiguity in Plato's Gorgias. Philosophy and Rhetoric, 41, pp. 212-237, 2008.

BEVERSLUIS, J. Cross-Examining Socrates. A Defense of the Interlocutors in Plato's Early Dialogues. Cambridge: Cambridge University Press, 2000.

BUZZETTI, E. The Injustice of Callicles and the Limits of Socrates's Ability to Educate a Young Politician. Ancient Philosophy, 25, pp. 25-63, 2005.

COULTER, J. A. The Relation of the Apology of Socrates to Gorgias Defense of Palamedes and Plato's Critique of Gorgianic Rhetoric. In: ERICKSON, K. V. Plato: True and Sophistic Rhetoric, Amsterdam: Rodopi, pp. 31-69 (réimpr. de l'article initialement publié dans les Harvard Studies in Classical Philology, 68, pp. 269-303, 1964).

DODDS, E. R. Plato. Gorgias. A Revised Text with Introduction and Commentary. Oxford: Clarendon Press, 1959.

EUBEN, J. P. Reading Democracy: "Socratic" Dialogues and the Political Education of Democratic Citizens. In: OBER, J., HEDRICK, C. Dêmokratia. Princeton: Princeton University Press, pp. 327-359, 1996.

FOUCAULT, M. Le gouvernement de soi et des autres. Cours au College de France (1982-1983). Édition établie, sous la direction de EWALD, F., FONTANA, A., par GROS, F. Paris: Gallimard-Le Seuil, 2008.

FRIEDLÄNDER, P. Gorgias. In: ERICKSON, K. V. Plato: True and Sophistic Rhetoric. Amsterdam: Rodopi, pp. 91-127, 1979 (reprise de FRIEDLÄNDER, P. Plato 2, The Dialogues. First Period. trad. de l'allemand par H. Meyerhoff, New York: Bollingen Series LIX, Pantheon Books, 1964, pp. 244-272).

GAUTHIER, R. A., JOLIF, J. Y. L'Éthique à Nicomaque, Introduction, traduction et commentaire. Louvain-Paris: Publications Universitaires de Louvain-Éditions Béatrice Nauwelaerts, 4 vol., 1970²; réimpr. Louvain-la-Neuve-Paris-Sterling, Virginia: Éditions Peeters, 2002. 
HAUSER, G. A. Aristotle on Epideictic: The Formation of Public Morality. Rhetoric Society Quarterly, 29, p. 5-23, 1999.

JEANMART, G. Généalogie de la docilité: dans l'Antiquité et le Haut Moyen Âge. Paris: Vrin, 2007.

KAHN, CH. H. Drama and Dialectic in Plato's Gorgias. Oxford Studies in Ancient Philosophy, 1, pp. 75-121, 1983.

MCADON, B. Reconsidering the Intention or Purpose of Aristotle's Rhetoric. Rhetoric Review, 23, p. 226-228, 2004.

MCCOY, M. Plato on the Rhetoric of Philosophers and Sophists. Cambridge: Cambridge University Press, 2008.

MCKIM, R. Shame and Truth in Plato's Gorgias. In GRISWOLD, CH. L. Platonic Writings. Platonic Readings. New York-London: Routledge, pp. 34-48, 1988.

MARKOVITS, E. The Politics of Sincerity: Plato, Frank Speech and Democratic Judgment. Penn State Press, 2008.

MONOSON, S.S. Plato's Democratic Entanglements: Athenian Politics and the Practice of Philosophy. Princeton: Princeton University Press, 2000.

NARCY, M. Ettre de bon conseil et savoir écouter (Éthique à Nicomaque, VI, 10-11). In: CHATEAU, J.Y. La vérité pratique: Aristote, Éthique à Nicomaque, Livre VI, Paris: Vrin, p. 117-135, 1997.

ROCCO, C. Liberating Discourse: The Politics of Truth in Plato's Gorgias. Interpretation, 23, pp. 361-385, 1996.

ROOCHNIK, D. Socrates Rhetorical Attack on Rhetoric. In: GONZALEZ, F. J. The Third Way. New Directions in Platonic Studies. Boston-London: Rowman \& Littlefield Publishers, pp. 81-94, 1995.

SAXONHOUSE, A. W. Free Speech and Democracy in Ancient Athens. Cambridge: Cambridge University Press, 2006.

SCHOFIELD, M. in Plato. Political Philosophy. Oxford: Oxford University Press, 2006. 
STALLEY, R. F. The Politics of the Gorgias. In: ERLER, M., BRISSON, L. Gorgias Menon, Selected Papers from the Seventh Symposium Platonicum. Sankt Augustin: Academia Verlag, pp. 116-121, 2007.

VAN RAALTE M. Socratic Parrhesia and its Afterlife in Plato's Laws. In: SLUITER, I., ROSEN, R. M., Free Speech in Classical Antiquity. Leiden-Boston: Brill, pp. 279-312, 2004.

WOERTHER, F. Lèthos aristotélicien, genèse d'une notion rhétorique. Paris: Vrin, 2007.

WOOLF, R. Callicles and Socrates: Psychic (Dis)harmony in the Gorgias. Oxford Studies in Ancient Philosophy,. 18, pp. 1-40, 2000.

E-mail: annie.hourcade@univ-rouen.fr

Recebido: julho/2009

Aprovado: agosto/2009 